University of Texas at El Paso

ScholarWorks@UTEP

\title{
On Decision Making Under Interval Uncertainty: A New Justification of Hurwicz Optimism-Pessimism Approach and its Use in Group Decision Making
}

\author{
Van Nam Huynh \\ Chenyi Hu \\ Yoshiteru Nakamori \\ Vladik Kreinovich \\ The University of Texas at El Paso, vladik@utep.edu
}

Follow this and additional works at: https://scholarworks.utep.edu/cs_techrep

Part of the Computer Engineering Commons

Comments:

Technical Report UTEP-CS-07-13

Published in the Proceedings of the 39th International Symposium on Multiple-Valued Logic ISMVL'2009, Naha, Okinawa, Japan, May 21-23, 2009, pp. 214-220.

\section{Recommended Citation}

Huynh, Van Nam; Hu, Chenyi; Nakamori, Yoshiteru; and Kreinovich, Vladik, "On Decision Making Under Interval Uncertainty: A New Justification of Hurwicz Optimism-Pessimism Approach and its Use in Group Decision Making" (2007). Departmental Technical Reports (CS). 107.

https://scholarworks.utep.edu/cs_techrep/107

This Article is brought to you for free and open access by the Computer Science at ScholarWorks@UTEP. It has been accepted for inclusion in Departmental Technical Reports (CS) by an authorized administrator of ScholarWorks@UTEP. For more information, please contact Iweber@utep.edu. 


\section{On Decision Making under Interval Uncertainty: A New Justification of Hurwicz Optimism-Pessimism Approach and Its Use in Group Decision Making}

\author{
Van Nam Huynh and \\ Yoshiteru Nakamori \\ Japan Advanced Institute of \\ Science and Technology (JAIST) \\ Nomi, Ishikawa 923-1292, Japan \\ huynh@jaist.ac.jp
}

\author{
Chenyi $\mathrm{Hu}$ \\ Computer Science Dept. \\ Univ. of Central Arkansas \\ Conway, AR 72035, USA \\ chu@uca.edu
}

\author{
Vladik Kreinovich \\ Dept. of Computer Science \\ Univ. of Texas at El Paso \\ El Paso, TX 79968, USA \\ vladik@utep.edu
}

\begin{abstract}
If we know the exact consequences of each action, then we can select an action with the largest value of the objective function. In practice, we often only know these values with interval uncertainty. If two intervals intersect, then some people may prefer the alternative corresponding to the first interval, and some prefer the alternative corresponding to the second interval. How can we describe the portion of people who select the first alternative? In this paper, we provide a new theoretical justification for Hurwicz optimism-pessimism approach, and we show how this approach can be used in group decision making.
\end{abstract}

\section{Introduction}

In many practical situations, the relative quality of different future situations can be described by a well-defined objective function: the larger the value of the objective function, the better the situation. For example, in the investment situations, the value of the investment at a certain future date is the objective function characterizing different situations: the larger this value, the better.

In these terms, if we have to decide between different situations, we should select the situation with the largest value of the corresponding objective function.

This same approach can be used when we decide between different possible actions. In the ideal case when we know the exact consequences of each action $a_{i}$, we can therefore predict the situation that arises from each action, and estimate the value $v_{i}$ of the objective function corresponding to this situation. In this ideal case, an action $a_{i}$ with the larger value $v_{i}$ is preferable to the action $a_{j}$ with a smaller value $v_{j}$. If we have to decided between different actions $a_{1}, \ldots, a_{n}$, then we select the action $a_{i}$ with the largest possible value of $v_{i}$.

In practice, we rarely know the exact consequences of different actions $a_{i}$. In this case, we do not know the exact value $v_{i}$ of the objective function; at best, we know the bounds $\underline{v}_{i}$ and $\bar{v}_{i}$ for this value: $\underline{v}_{i} \leq v_{i} \leq \bar{v}_{i}$. The first natural question is: under this uncertainty, how should we select an appropriate action?

One of the ways to select such an action is known as the Hurwicz optimism-pessimism approach: we find a value $\alpha \in[0,1]$ that describe the user's degree of optimism, and select the alternative for which the value $\alpha \cdot \bar{v}_{i}+(1-\alpha) \cdot \underline{v}_{i}$ is the largest possible.

There are some justifications for this approach, but researchers do not consider these justifications fully satisfactory. In this paper, we provide a new (hopefully, more satisfactory) justification for the Hurwicz approach.

The next natural question is: how to combine preferences of different users, users that have different degrees of optimism? This problem is also analyzed in this paper.

\section{Formulation of the Problem}

Decision making in the absence of uncertainty. Often, in decision making, we know what quantity we want to maximize (or minimize) under certain constraints. In other words, we know the objective function that we want to maximize (or minimize). For example, in running a business, we usually maximize profits - within given social and environmental constraints. When planning a trip by car, we usually minimize the travel time - within given constraints describing convenience etc. When planning a trip by plane, many of us minimize the travel cost, etc. 
As an example, let us assume that we have two alternatives $a_{1}$ and $a_{2}$. Let us also assume that we know the exact values $v_{1}$ and $v_{2}$ of the (maximized) objective function for these alternatives: if we select the alternative $a_{1}$, then the value of the objective function characterizing the user's preference is $v_{1}$; and if we select the alternative $a_{2}$, then the value of the objective function characterizing the user's preference is $v_{2}$.

In this situation, the decision is easy:

- if $v_{1}>v_{2}$, we should select alternative $a_{1}$;

- if $v_{1}<v_{2}$, we should select alternative $a_{2}$;

- if $v_{1}=v_{2}$, we can select any of the two alternatives, the result will be the same.

Case of interval uncertainty. In practice, we usually do not know the exact values $v_{i}$ of the objective function - because we do not know the exact state of the world. For example, the actual future profit of an insurance company will depend on whatever natural disasters happen.

Often, because of the imcompeleteness of our knowledge, we only know the interval $\left[\underline{v}_{i}, \bar{v}_{i}\right]$ of possible values of $v_{i}$. How can we then make a decision?

Sometimes, decision is straightforward even under interval uncertainty. If $\bar{v}_{1} \leq \underline{v}_{2}$, then it can be guaranteed that the (unknown) actual values $v_{1} \in\left[\underline{v}_{1}, \bar{v}_{1}\right]$ and $v_{2} \in\left[\underline{v}_{2}, \bar{v}_{2}\right]$ satisfy the inequality $v_{1} \leq v_{2}$. Indeed, in this case, $v_{1} \leq \bar{v}_{1}, \bar{v}_{1} \leq \underline{v}_{2}$, and $\underline{v}_{2} \leq v_{2}$; thus, $v_{1} \leq v_{2}$. So, in this case, the second alternative $a_{2}$ is always better (or at least of the same quality) as $a_{1}$. Thus, in this case, a decision maker should select $a_{2}$.

In should be mentioned that in the particular subcase of this case, when $\bar{v}_{1}=\underline{v}_{2}$, it is possible that $v_{1}=\bar{v}_{1}$ and $v_{2}=\underline{v}_{2}$. In this subcase, $v_{1}=v_{2}$ and thus, selecting $a_{1}$ also leads to the optimal solution.

Similarly, if $\bar{v}_{2} \leq \underline{v}_{1}$, then it can be guaranteed that the (unknown) actual values $v_{1} \in\left[\underline{v}_{1}, \bar{v}_{1}\right]$ and $v_{2} \in\left[\underline{v}_{2}, \bar{v}_{2}\right]$ satisfy the inequality $v_{2} \leq v_{1}$. Indeed, in this case, $v_{2} \leq \bar{v}_{2}$, $\bar{v}_{2} \leq \underline{v}_{1}$, and $\underline{v}_{1} \leq v_{1}$; thus, $v_{2} \leq v_{1}$. So, in this case, the first alternative $a_{1}$ is always better (or at least of the same quality) as $a_{2}$. Thus, in this case, a decision maker should select $a_{1}$.

But what if the intervals $\left[\underline{v}_{1}, \bar{v}_{1}\right]$ and $\left[\underline{v}_{2}, \bar{v}_{2}\right]$ have a common non-degenerate subinterval? In this case, we may have $v_{1}<v_{2}$ and we may also have $v_{2}<v_{1}$. Which of the alternatives should we choose?

Our first result: individual decision making. In this paper, we first show that if we want to be able to always make a selection, a selection that should not depend on the choice of the measuring unit or on the choice of the starting point, then we should use a Hurwicz criterion, i.e., fix a value $\alpha \in[0,1]$ and then select an alternative $a_{i}$ for which the value $\alpha \cdot \bar{v}_{i}+(1-\alpha) \cdot \underline{v}_{i}$ is the largest possible $[4,5]$. It is known that different values of $\alpha$ represent different human behaviors:

- the value $\alpha=1$ means that when we make a decision, we only take into account the best possible outcome $\bar{v}_{i}$; this risk-prone behavior corresponds to optimism;

- the value $\alpha=0$ means that when we make a decision, we only take into account the worst possible outcome $\underline{v}_{i}$; this risk-averse behavior corresponds to pessimism;

- values $\alpha \in(0,1)$ mean that we take into account both the best and the worst possible outcomes; the corresponding value $\alpha$ describes the weight of the best possible outcome.

Our second result: group decision making. In group decision making, we have people with different behaviors. So, if we have two alternatives $a_{1}$ and $a_{2}$, different people will select different alternatives. In such a situation, the portion of people who selected $a_{1}$ is a reasonable indication of how better $a_{1}$ is for this group. In other words, instead of a single yes-no answer ( $a_{1}$ is better or $a_{2}$ is better), we should generate a number $p\left(a_{1}>a_{2}\right)$ characterizing the degree to which $a_{1}$ is better than $a_{2}$. In this paper, we will derive such a formula.

Individual decision making under interval uncertainty: a new justification of Hurwicz criterion. We need to have a linear (= total) (pre-)order $\preceq$ on the set of all intervals (degenerate or non-degenerate), i.e., an order in which for every two intervals $\mathbf{v}_{1}=\left[\underline{v}_{1}, \bar{v}_{1}\right]$ and $\mathbf{v}_{2}=\left[\underline{v}_{2}, \bar{v}_{2}\right]$, we should have $\mathbf{v}_{1} \preceq \mathbf{v}_{2}$ or $\mathbf{v}_{2} \preceq \mathbf{v}_{1}$. When $\bar{v}_{1} \leq \underline{v}_{2}$, we should have $\mathbf{v}_{1} \preceq \mathbf{v}_{2}$.

Another reasonable requirement is that if $\mathbf{v}_{n} \preceq \mathbf{v}_{n}^{\prime}$ for all $n$, then in the limit $n \rightarrow \infty$, when $\mathbf{v}_{n} \rightarrow \mathbf{v}$ and $\mathbf{v}_{n}^{\prime} \rightarrow \mathbf{v}^{\prime}$, we should also have $\mathbf{v} \preceq \mathbf{v}^{\prime}$. Indeed, from the practical viewpoint, the fact that $\mathbf{v}_{n} \rightarrow \mathbf{v}$ means that whatever accuracy we choose, when $n$ is large enough, then $\mathbf{v}_{n}$ is indistinguishable from $\mathbf{v}$ within this accuracy. In practice, we always estimate consequences of our actions with some uncertainty. Thus, in practice, when $n$ is large enough, $\mathbf{v}$ is indistinguishable from $\mathbf{v}_{n}$ and $\mathbf{v}^{\prime}$ from $\mathbf{v}_{n}^{\prime}$. Since $\mathbf{v}_{n} \preceq \mathbf{v}_{n}^{\prime}$, we can thus conclude that $\mathbf{v} \preceq \mathbf{v}^{\prime}$.

It is also reasonable to require that the order should not depend on the choice of a starting value or a measuring unit for measuring $v$. For example, when we compare intervals for profits, the results of this comparison should not change whether we count profit in dollars or in euros, and whether we compare profits themselves or differences between the 
profit and some predicted value $v_{0}$. In precise terms, changing a starting point from 0 to $v_{0}$ means subtracting $v_{0}$, and changing a unit to a new one which is $\lambda$ times smaller means multiplying all numerical values by $\lambda$. Thus, we arrive at the following definitions.

Definition 1. By an interval order, we mean a transitive symmetric relation $\preceq$ on the set of all (degenerate or nondegenerate) intervals for which:

- for every two intervals $\mathbf{v}_{1}$ and $\mathbf{v}_{2}$, either $\mathbf{v}_{1} \preceq \mathbf{v}_{2}$ or $\mathbf{v}_{2} \preceq \mathbf{v}_{1}$ (or both);

- if $\bar{v}_{1} \leq \underline{v}_{2}$, then $\left[\underline{v}_{1}, \bar{v}_{1}\right] \preceq\left[\underline{v}_{2}, \bar{v}_{2}\right]$;

- if $\bar{v}_{1}<\underline{v}_{2}$, then $\left[\underline{v}_{2}, \bar{v}_{2}\right] \npreceq\left[\underline{v}_{1}, \bar{v}_{1}\right]$.

Definition 2. We say that an interval order is closed if $\mathbf{v}_{n} \preceq$ $\mathbf{v}_{n}^{\prime}, \mathbf{v}_{n} \rightarrow \mathbf{v}$, and $\mathbf{v}_{n}^{\prime} \rightarrow \mathbf{v}^{\prime}$ imply that $\mathbf{v} \preceq \mathbf{v}^{\prime}$.

Definition 3. We say that an interval order is shift- and scale-invariant iffor every $v_{0}$ and $\lambda>0,\left[\underline{v}_{1}, \bar{v}_{1}\right] \preceq\left[\underline{v}_{2}, \bar{v}_{2}\right]$ implies

$$
\left[\lambda \cdot \underline{v}_{1}+v_{0}, \lambda \cdot \bar{v}_{1}+v_{0}\right] \preceq\left[\lambda \cdot \underline{v}_{2}+v_{0}, \lambda \cdot \bar{v}_{2}+v_{0}\right] .
$$

Proposition 1. For every closed shift- and scale-invariant interval order, there exists a constant $\alpha \in[0,1]$ such that $\left[\underline{v}_{1}, \bar{v}_{1}\right] \preceq\left[\underline{v}_{2}, \bar{v}_{2}\right]$ if and only if

$$
\alpha \cdot \bar{v}_{1}+(1-\alpha) \cdot \underline{v}_{1} \leq \alpha \cdot \bar{v}_{2}+(1-\alpha) \cdot \underline{v}_{2} .
$$

Discussion. In other words, every closed and invariant interval order can be described by some Hurwicz criterion. Vice versa, for every $\alpha$, the corresponding interval order is closed and invariant. Thus, for individual decision making, we should use Hurwicz criterion.

Comment. It is worth mentioning that there exist alternative justifications of Hurwicz criterion; see, e.g., [6, 8].

Mathematical comment. Strictly speaking, for every $\alpha \in$ $[0,1]$, the corresponding relation

$$
\alpha \cdot \bar{v}_{1}+(1-\alpha) \cdot \underline{v}_{1} \leq \alpha \cdot \bar{v}_{2}+(1-\alpha) \cdot \underline{v}_{2}
$$

between intervals $\left[\underline{v}_{1}, \bar{v}_{1}\right]$ and $\left[\underline{v}_{2}, \bar{v}_{2}\right]$ is a pre-order, not an order. Note that, for an order, $a \preceq b$ and $b \preceq a$ imply that $a=b$. Let us show that for the above relation, this is not true for the intervals $\left[\underline{v}_{1}, \bar{v}_{1}\right]=[0,1]$ and $\left[\underline{v}_{2}, \bar{v}_{2}\right]=[\alpha, \alpha]$. Indeed, for these intervals, we have

$$
\alpha \cdot \bar{v}_{1}+(1-\alpha) \cdot \underline{v}_{1}=\alpha \cdot 1+(1-\alpha) \cdot 0=\alpha
$$

and

$$
\alpha \cdot \bar{v}_{2}+(1-\alpha) \cdot \underline{v}_{2}=\alpha \cdot \alpha+(1-\alpha) \cdot \alpha=\alpha .
$$

Thus, for these two intervals, we have

$$
\alpha \cdot \bar{v}_{1}+(1-\alpha) \cdot \underline{v}_{1} \leq \alpha \cdot \bar{v}_{2}+(1-\alpha) \cdot \underline{v}_{2},
$$

i.e., $\left[\underline{v}_{1}, \bar{v}_{1}\right]=[0,1] \preceq[\alpha, \alpha]=\left[\underline{v}_{2}, \bar{v}_{2}\right]$.

Similarly, we have

$$
\alpha \cdot \bar{v}_{2}+(1-\alpha) \cdot \underline{v}_{2} \leq \alpha \cdot \bar{v}_{1}+(1-\alpha) \cdot \underline{v}_{1},
$$

i.e., $\left[\underline{v}_{2}, \bar{v}_{2}\right]=[\alpha, \alpha] \preceq[0,1]=\left[\underline{v}_{1}, \bar{v}_{1}\right]$. Thus, we have $[0,1] \preceq[\alpha, \alpha]$ and $[\alpha, \alpha] \preceq[0,1]$, but $[0,1] \neq[\alpha, \alpha]$.

Proof. Let $\preceq$ be a closed invariant interval order.

$1^{\circ}$. Let us first consider degenerate intervals. For degenerate intervals $[x, x]$ (i.e., real numbers $x$ ) the definition of an interval order implies that if $x \leq x^{\prime}$ then $[x, x] \preceq\left[x^{\prime}, x^{\prime}\right]$. So, if $x \leq x^{\prime}$ in the sense of the normal ordering between real numbers, then we also have $x \preceq x^{\prime}$ in the sense of the interval order.

Similarly, if $x \not \leq x^{\prime}$ in the sense of the normal order between real numbers, i.e., if $x>x^{\prime}$, then, according to the definition of an interval order, we should have $[x, x] \npreceq$ $\left[x^{\prime}, x^{\prime}\right]$. So, if $x \not \leq x^{\prime}$ in the sense of the normal ordering between real numbers, then we also have $x \npreceq x^{\prime}$ in the sense of the interval order.

Thus, on real numbers, the interval order coincides with the usual one.

$2^{\circ}$. Let us now start extending this order to non-degenerate intervals by considering the simplest non-degenerate interval, e.g., the interval $[0,1]$.

What can we say about the relation between this interval and real numbers? Let $S$ denote the set of all the values $x \geq 0$ for which $[x, x] \preceq[0,1]$. By definition of an interval order, $[0,0] \preceq[0,1]$ (i.e., $0 \in S$ ) and $[x, x] \npreceq[0,1]$ when $x>1$, i.e., $S \subseteq[0,1]$. Let us denote the supremum of the set $S$ by $\alpha$.

By definition of the supremum, every element of $S$ is $\leq \alpha$, and for every $\varepsilon>0$, there exists a value $x_{n}>\alpha-\varepsilon$ for which $x_{n} \in S$, i.e., for which $x_{n} \preceq[0,1]$. In the limit $\varepsilon \rightarrow 0$, from $\alpha-\varepsilon \leq x_{n} \leq \alpha$, we conclude that $x_{n} \rightarrow \alpha$. Thus, from the fact that $\leq$ is closed, we conclude that $\alpha \preceq$ $[0,1]$.

Since $\alpha$ is the supremum, for every $\varepsilon>0$, we have $\alpha+$ $\varepsilon \notin S$, i.e., $\alpha+\varepsilon \npreceq[0,1]$. By definition, an interval preorder, if $a \npreceq b$, then $b \preceq a$. So, we conclude that $[0,1] \preceq$ $\alpha+\varepsilon$. In the limit $\varepsilon \rightarrow 0$, we get $[0,1] \preceq \alpha$.

So, we have both $\alpha \preceq[0,1]$ and $[0,1] \preceq \alpha$, hence $[0,1] \sim \alpha$ (where the equivalence relation $a \sim b$ means $a \preceq b$ and $b \preceq a$ ).

$3^{\circ}$. Now, we are ready to handle arbitrary non-degenerate intervals. 
For every non-degenerate interval $[\underline{v}, \bar{v}]$, we have $[\underline{v}, \bar{v}]=$ $\left[\lambda \cdot 0+v_{0}, \lambda \cdot 1+v_{0}\right]$, for $\lambda=\bar{v}-\underline{v}$ and $v_{0}=\underline{v}$. Thus, using the invariance of $\preceq$, we conclude that

$$
[\underline{v}, \bar{v}] \sim \lambda \cdot \alpha+v_{0}=\lambda \cdot(\bar{v}-\underline{v})+\underline{v} .
$$

This expression is exactly equal to Hurwicz's expression $\alpha$. $\bar{v}+(1-\alpha) \cdot \underline{v}$. Thus, each interval is equivalent to its Hurwicz value.

Since for real numbers, the interval order coincides with the standard order between real numbers, we conclude that an interval $\mathbf{v}_{2}$ is "preferable" (in the sense of the interval order) than $\mathbf{v}_{1}$ if and only if the Hurwicz value corresponding to $\mathbf{v}_{2}$ is larger than the Hurwicz value corresponding to $\mathbf{v}_{1}$.

The proposition is proven.

\section{Towards Group Decision Making under In- terval Uncertainty}

Formulation of the problem. In the previous section, we have shown that rational individual decision making under interval uncertainty should be following Hurwicz criterion for some $\alpha$. In a group, we can have individuals with different values $\alpha$. For given two intervals, some may select $\mathbf{v}_{1}$, some may select $\mathbf{v}_{2}$, and some may select both (if for them the corresponding Hurwicz values coincide). What is the portion of people selecting $\mathbf{v}_{2}$ ?

Cases when answer is clear. In some situations, the answer is clear. For example, if $\underline{v}_{1} \leq \underline{v}_{2}$ and $\bar{v}_{1} \leq \bar{v}_{2}$, then we have $\alpha \cdot \bar{v}_{1}+(1-\alpha) \cdot \underline{v}_{1} \leq \alpha \cdot \bar{v}_{2}+(1-\alpha) \cdot \underline{v}_{2}$ for every $\alpha \in[0,1]$. So, in this case, all individuals will select $\left[\underline{v}_{2}, \bar{v}_{2}\right]$, i.e., the portion is 1 .

Cases when answer is not straightforward. In other cases, e.g., when $\underline{v}_{2}<\underline{v}_{1}<\bar{v}_{1}<\bar{v}_{2}$, the selection depends on $\alpha$ : for an optimist $\alpha=1, \mathbf{v}_{2}$ is better, but for a pessimist, $\mathbf{v}_{1}$ is better. In such situations, the desired portion depends on the distribution of values $\alpha$.

\subsection{Setting}

It is reasonable to describe this distribution by using a (cumulative) distribution function. Specifically, for every $\alpha_{0}$, let $F(\alpha)$ denote the portion of individuals for whom $\alpha \leq \alpha_{0}$, and let $F^{-}(\alpha)$ denote the portion of individuals for whom $\alpha<\alpha_{0}$. In these terms, the portion of individuals for whom $\alpha \geq \alpha_{0}$ is equal to $1-F^{-}\left(\alpha_{0}\right)$.

Proposition 2. Let $F(\alpha)$ be a cumulative distribution function on the interval $[0,1]$, and let $\mathbf{v}_{1}=\left[\underline{v}_{1}, \bar{v}_{1}\right]$ and $\mathbf{v}_{2}=\left[\underline{v}_{2}, \bar{v}_{2}\right]$ be two intervals of widths $w_{i} \stackrel{\text { def }}{=} \bar{v}_{i}-\underline{v}_{i}$.
Then, the probability $P_{2}$ that

$$
\alpha \cdot \bar{v}_{1}+(1-\alpha) \cdot \underline{v}_{1} \leq \alpha \cdot \bar{v}_{2}+(1-\alpha) \cdot \underline{v}_{2}
$$

is equal to the following:

- When $w_{1}<w_{2}$, we have $P_{2}=1$ if $\underline{v}_{1} \leq \underline{v}_{2}, P_{2}=0$ if $\bar{v}_{2}<\bar{v}_{1}$, and otherwise $P_{2}=1-F^{-}(t)$, where

$$
t \stackrel{\text { def }}{=} \frac{\underline{v}_{1}-\underline{v}_{2}}{w_{2}-w_{1}}
$$

- When $w_{1}>w_{2}$, we have $P_{2}=1$ if $\bar{v}_{2} \geq \bar{v}_{1}, P_{2}=0$ if $\underline{v}_{2}<\underline{v}_{1}$, and otherwise $P_{2}=F(t)$.

- When $w_{1}=w_{2}$, we have $P_{2}=1$ if $\underline{v}_{1} \leq \underline{v}_{2}$ and $P_{2}=0$ if $\underline{v}_{1} \leq \underline{v}_{2}$.

Proof. If we open parentheses in the inequality $\alpha \cdot \bar{v}_{1}+$ $(1-\alpha) \cdot \underline{v}_{1} \leq \alpha \cdot \bar{v}_{2}+(1-\alpha) \cdot \underline{v}_{2}$ and move all the terms proportional to $\alpha$ to one side, we get an equivalent inequality

$$
\alpha \cdot\left(\left(\bar{v}_{2}-\underline{v}_{2}\right)-\left(\bar{v}_{2}-\underline{v}_{1}\right)\right) \geq \underline{v}_{1}-\underline{v}_{2},
$$

or, equivalently,

$$
\alpha \cdot\left(w_{2}-w_{1}\right) \geq \underline{v}_{1}-\underline{v}_{2} .
$$

Let us consider all three cases from the formulation of the Proposition: $\left.w_{2}>w_{1}, w\right) 1<w_{2}$, and $w_{1}=w_{2}$.

Let us first consider the case $w_{2}>w_{1}$. In this case, the inequality for $\alpha$ is equivalent to

$$
\alpha \geq t \stackrel{\text { def }}{=} \frac{\underline{v}_{1}-\underline{v}_{2}}{w_{2}-w_{1}} .
$$

We will consider three subcases: $t \leq 0, t>1$, and $0<t \leq$ 1 .

The first subcase is $t \leq 0$. By definition of $t$, taking into account that we are in the case $w_{2}>w_{1}$, we conclude that the inequality $t \leq 0$ is equivalent to $\underline{v}_{1} \leq \underline{v}_{2}$. When $t \leq 0$, then then the inequality $\alpha \geq t$ holds for all $\alpha$. Thus, the probability $P_{2}$ that this inequality is satisfied is equal to 1 .

The second subcase if $t>1$. Multiplying both sides of the inequality $t>1$ by the positive value $w_{2}-w_{1}$, we conclude that $t>1$ is equivalent to $\underline{v}_{1}-\underline{v}_{2}>w_{2}-w_{1}=$ $\left(\bar{v}_{2}-\underline{v}_{2}\right)-\left(\bar{v}_{1}-\underline{v}_{1}\right)$. Opening the parentheses and canceling terms $\underline{v}_{i}$ in both sides, we get an equivalent form $0>\bar{v}_{2}-$ $\bar{v}_{1}$, i.e., if $\bar{v}_{2}<\bar{v}_{1}$. When $t>1$, then the inequality $\alpha \geq t$ cannot hold for any $\alpha \in[0,1]$. Therefore, the probability $P_{2}$ that this inequality holds is equal to 0 .

The only remaining subcase is $0<t \leq 1$. In this subcase, the desired probability $P_{2}$ is the probability that $\alpha \geq t$, i.e., $P_{2}=1-F^{-}(t)$.

Similar formulas can be described for the case when $w_{1}<w_{2}$. 
When $w_{1}=w_{2}$, then for $\underline{v}_{1} \leq \underline{v}_{2}$ we have $P_{2}=1$, and for $\underline{v}_{1}>\underline{v}_{2}$ we have $P_{2}=0$. The proposition is proven.

Comment. One can see that the desired portion monotonically depends on the quantity $t$. In $[2,3]$, the "degree of selection" $d$ is defined as (in our notations) $d \stackrel{\text { def }}{=} \frac{\bar{v}_{2}-\bar{v}_{1}}{2\left(w_{2}-w_{1}\right)}$. One can easily see that $t+2 d=1$, hence $t=1-2 d$, $d=(1-t) / 2$, and monotonic dependence on $t$ means exactly monotonic dependence on $d$. Thus, we have justified the use of the empirical expression $d$.

In particular, for the case when $w_{1}<w_{2}$, and the distribution of $\alpha$ is uniform (i.e., $F(\alpha)=F^{-}(\alpha)=\alpha$ for all $\alpha$ ), the portion (when it is not equal to 0 or 1 ), is equal to $1-F^{-}(t)=1-t=2 d$.

Comment on selection between equal alternatives. In the above text, we deal with the portion $P_{1}$ of those who select $\mathbf{v}_{1}$, and with a portion $P_{2}$ of those who select $\mathbf{v}_{2}$. We have mentioned that those to whom $\mathbf{v}_{1}$ and $\mathbf{v}_{2}$ are equivalent are included in both counts, i.e., $P_{1}+P_{2} \geq 1$ and it is possible that $P_{1}+P_{2}>1$ : e.g., if $\mathbf{v}_{1}$ and $\mathbf{v}_{2}$ are identical, we have $P_{1}=P_{2}=1$ and $P_{1}+P_{2}=1$.

When we compute the sum $P_{1}+P_{2}$, those who selected only $\mathbf{v}_{1}$ or only $\mathbf{v}_{2}$ are counted exactly once, but those who selected both are counted twice. Thus, $P_{1}+P_{2}$ equals 1 plus the portion of those who selected both alternatives. Hence, the difference $P_{1}+P_{2}-1$ is the portion of those who selected both, and the remaining portions $P_{1}-\left(P_{1}+P_{2}-1\right)=1-P_{2}$ and $P_{2}-\left(P_{1}+P_{2}-1\right)=1-P_{1}$ described those who selected only $\mathbf{v}_{1}$ or only $\mathbf{v}_{2}$.

A reasonable alternative description is to assume that if for a person, two alternatives are equivalent, then this person will select one of them with probability $\frac{1}{2}$; see, e.g., $[2,3]$. In this case, the alternative $\mathbf{v}_{1}$ will be accepted in a portion

$$
1-P_{2}+\frac{P_{1}+P_{2}-1}{2}=\frac{1+P_{1}-P_{2}}{2},
$$

and the alternative $\mathbf{v}_{2}$ will be accepted in a portion

$$
1-P_{1}+\frac{P_{1}+P_{2}-1}{2}=\frac{1-P_{1}+P_{2}}{2} .
$$

\section{Auxiliary Result: Reasonable Distributions of Optimism Degree}

Formulation of a problem. In the previous text, we did not make any assumptions about the distribution function $F(\alpha)$, and we got formulas which explicitly depend on this distribution. A natural question is: which distribution functions $F(\alpha)$ are possible?

Idea. Let us consider a situation in which the only information we have about the value $v$ of the desired objective function at a given alternative is that this value belongs to the interval $[0,1]$. For a decision maker who uses Hurwicz criterion with the parameter $\alpha$, this uncertain situation is equivalent to using a single value $v=\alpha \cdot 1+(1-\alpha) \cdot 0=\alpha$. Thus, in this uncertain situation, we have different equivalent values $v$ ranging from 0 to 1 , and their distribution is characterized by the distribution function $F(\alpha)$. Here, the probability that $v \in[\underline{\alpha}, \bar{\alpha}]$ is equal to $F(\bar{\alpha})-F^{-}(\underline{\alpha})$.

Suppose now that we gained some additional information about the alternative, and because of this information, we now conclude that $v$ belongs to the narrower interval $[\underline{v}, \bar{v}] \subset[0,1]$. How can we describe the new distribution of equivalent values?

There are two possible approaches. First, we can simply equate the probability that $v \in[\underline{\alpha}, \bar{\alpha}]$ (where $[\underline{\alpha}, \bar{\alpha}] \subseteq[\underline{v}, \bar{v}]$ ) with the conditional probability - under the condition that $\alpha \in[\underline{v}, \bar{v}]$, i.e., with the value

$$
\frac{F(\bar{\alpha})-F^{-}(\underline{\alpha})}{F(\bar{v})-F^{-}(\underline{v})} .
$$

Alternatively, we can argue that now $\underline{v}$ is the new pessimistic estimate and $\bar{v}$ is the new optimistic estimate, so each value $v=\underline{v}+\alpha \cdot(\bar{v}-\underline{v})$ is distributed according to the distribution $F(\alpha)$. For each $v \in[\underline{v}, \bar{v}]$, the corresponding $\alpha$ can be computed from the condition that $v=\underline{v}+\alpha \cdot(\bar{v}-\underline{v})$; this $\alpha$ is equal to $\alpha=\frac{v-\underline{v}}{\bar{v}-\underline{v}}$. Thus, the probability to have $v \in[\underline{\alpha}, \bar{\alpha}]$ is equal to the probability that the optimismpessimism parameter $\alpha$ is in the interval

$$
\left[\frac{\underline{\alpha}-\underline{v}}{\bar{v}-\underline{v}}, \frac{\bar{\alpha}-\underline{v}}{\bar{v}-\underline{v}}\right]
$$

This probability is equal to

$$
F\left(\frac{\bar{\alpha}-\underline{v}}{\bar{v}-\underline{v}}\right)-F^{-}\left(\frac{\underline{\alpha}-\underline{v}}{\bar{v}-\underline{v}}\right) .
$$

It is reasonable to require that these two ways should lead to exact same formula for the probability. As a result, we arrive at the following definition:

Definition 4. By a distribution function for optimism degree (or simply distribution function, for short), we mean a monotonic function $F:[0,1] \rightarrow[0,1]$ for which $F(1)=1$.

Definition 5. For each distribution function $F(z)$, we define $F^{-}(\alpha)$ as follows: $F^{-}(0)=0$ and for $\alpha>0, F^{-}(\alpha)=$ $\sup \{F(z): z<\alpha\}$.

Definition 6. We say that a distribution function is consistent if for every three values $\underline{v} \leq v \leq \bar{v}$ from the interval $[0,1]$, we have

$$
\frac{F(\bar{\alpha})-F^{-}(\underline{\alpha})}{F(\bar{v})-F^{-}(\underline{v})}=F\left(\frac{\bar{\alpha}-\underline{v}}{\bar{v}-\underline{v}}\right)-F^{-}\left(\frac{\underline{\alpha}-\underline{v}}{\bar{v}-\underline{v}}\right) .
$$


Comment. This idea is similar to the one used in [7] in a similar situation.

Proposition 3. The only consistent distribution function for optimism degree is the function $F(\alpha)=\alpha$ corresponding to the uniform distribution of the interval $[0,1]$.

Comment. As we have mentioned, for this function $F$, the portions become proportional to formulas from $[2,3]$.

Proof. Let us first consider the case when $v=\alpha=0$, $\bar{v}=x$, and $\bar{\alpha}=x \cdot y$ for some $x, y \in[0,1]$. In this case, the consistency condition takes the form

$$
\frac{F(x \cdot y)-0}{F(x)}=F\left(\frac{x \cdot y}{x}\right)-0,
$$

i.e., the form $F(x \cdot y)=F(x) \cdot F(y)$. It is known (see, e.g., [1]), that the only monotonic solutions of this functional equation are the functions $F(x)=x^{k}$ for some $k>0$.

So, to complete the proof, it suffices to show that $k=1$. Indeed, let us now consider the case when $\underline{v}=\underline{\alpha}=1 / 2$, $\bar{v}=1$, and $\bar{\alpha}=\frac{1+z}{2}$ for some $z \in[0,1]$. In this case, the consistency condition takes the form

$$
\frac{\left(\frac{1+z}{2}\right)^{k}-\left(\frac{1}{2}\right)^{k}}{1^{k}-\left(\frac{1}{2}\right)^{k}}=z^{k} .
$$

Multiplying both the numerator and the denominator of the left-hand side by $2^{k}$, we get

$$
\frac{(1+z)^{k}-1}{2^{k}-1}=z^{k}
$$

This equality must be true for all $z \in[0,1]$. Differentiating by $z$ and taking $z=0$, we conclude that

$$
\frac{k}{2^{k}-1}=k \cdot 0^{k-1}
$$

The left-hand side of this limit equality is finite and positive, the right-hand side is 0 for $k>1$ and infinite for $k<1$. Thus, the only possible value is $k=1$. The proposition is proven.

\section{Conclusions and Future Work}

Conclusions. In many practical situations, we do not know the exact consequences of different actions. As a result, for each action $a_{i}$, we do not know the exact value $v_{i}$ of the corresponding objective function; we only know the bounds $\underline{v}_{i}$ and $\bar{v}_{i}$ on this value: $v_{i} \in\left[\underline{v}_{i}, \bar{v}_{i}\right]$. Under this interval uncertainty, which alternative $a_{i}$ should we select?

In this paper, we have shown that for this selection, it is reasonable to use Hurwicz optimism-pessimism approach: namely,

- choose a value $\alpha \in[0,1]$, and

- select an alternative $a_{i}$ with the largest possible value of the quantity $\alpha \cdot \bar{v}_{i}+(1-\alpha) \cdot \underline{v}_{i}$.

The value $\alpha$ describes the user's degree of optimism.

We have also analyzed what will happen if the decision must be made a group, and different members of the group have different degrees of optimism.

- Once we know the probabilities of different values of $\alpha$, we can then describe the probability of selecting different alternatives.

- We have also shown that if we have no information about the probability distribution of $\alpha$, it is reasonable to assume that these values are uniformly distributed on the interval $[0,1]$.

Future work. In this paper, we considered two extreme cases: when we know the probability distribution of $\alpha$, and when we have no information about this probability distribution at all. In practice, we sometimes have partial information about this probability distribution. In the future, it is desirable to consider group decision making under such partial information.

Acknowledgments. This work was partially supported: by NSF Grants CCF-0202042, EAR-0225670, and HRD0734825, by Grant 1 T36 GM078000-01 from the National Institutes of Health, by Texas Department of Transportation grant No. 0-5453, and by the Japan Advanced Institute of Science and Technology (JAIST) International Joint Research Grant 2006-08.

The authors are thankful to the anonymous referees for the valuable suggestions.

\section{References}

[1] J. Aczel, Lectures on functional equations and their applications, Dover, New York, 2006.

[2] P. Hu, M. Deallar, and C. Hu, "Task scheduling on flow networks with temporal uncertainty", Proceedings of the 2007 IEEE Symposium on Foundations of Computational Intelligence FOCI'07, Honolulu, Hawaii, April 1-5, 2007, to appear. 
[3] P. Hu and C. Hu, "Fuzzy partial-order relations for intervals and interval-weighted graphs", Proceedings of the 2007 IEEE Symposium on Foundations of Computational Intelligence FOCI'07, Honolulu, Hawaii, April 1-5, 2007, to appear.

[4] L. Hurwicz, A criterion for decision-making under uncertainty, Technical Report 355, Cowles Commission, 1952.

[5] R. D. Luce and H. Raiffa, Games and Decisions, Dover, New York, 1989.

[6] H. T. Nguyen and V. Kreinovich, "Nested Intervals and Sets: Concepts, Relations to Fuzzy Sets, and Applications", In: R. B. Kearfott et al (eds.), Applications of Interval Computations, Kluwer, Dordrecht, 1996, pp. 245-290.
[7] H. T. Nguyen, V. Kreinovich, and L. Longpré, "Dirty Pages of Logarithm Tables, Lifetime of the Universe, and (Subjective) Probabilities on Finite and Infinite Intervals", Reliable Computing, 2004, Vol. 10, No. 2, pp. 83-106.

[8] H. T. Nguyen, V. Kreinovich, and Q. Zuo, "Intervalvalued degrees of belief: applications of interval computations to expert systems and intelligent control", International Journal of Uncertainty, Fuzziness, and Knowledge-Based Systems (IJUFKS), 1997, Vol. 5, No. 3, pp. 317-358. 